Sādhanā Vol. 40, Part 2, April 2015, pp. 591-604. (C) Indian Academy of Sciences

\title{
Flexural vibrations of finite composite poroelastic cylinders
}

\author{
SANDHYA RANI BANDARI*, SRISAILAM ALETI and \\ MALLA REDDY PERATI
}

Department of Mathematics, Kakatiya University, Warangal 506 009, India

e-mail: sandhyarani.bandari@gmail.com; asmou22@yahoo.com;

mperati@yahoo.com

MS received 10 April 2014; revised 27 October 2014; accepted 12 December 2014

\begin{abstract}
This paper deals with the flexural vibrations of composite poroelastic solid cylinder consisting of two cylinders that are bonded end to end. Poroelastic materials of the two cylinders are different. The frequency equations for pervious and impervious surfaces are obtained in the framework of Biot's theory of wave propagation in poroelastic solids. The gauge invariance property is used to eliminate one arbitrary constant in the solution of the problem. This would lower the number of boundary conditions actually required. If the wavelength is infinite, frequency equations are degenerated as product of two determinants pertaining to extensional vibrations and shear vibrations. In this case, it is seen that the nature of the surface does not have any influence over shear vibrations unlike in the case of extensional vibrations. For illustration purpose, three composite cylinders are considered and then discussed. Of the three, two are sandstone cylinders and the third one is resulted when a cylindrical bone is implanted with Titanium. In either case, phase velocity is computed against aspect ratios.
\end{abstract}

Keywords. Flexural vibrations; finite composite cylinder; poroelasticity; frequency equation; phase velocity; aspect ratio.

\section{Introduction}

We know from daily experience that many man-made structures consist of two or more than two parts bonded end to end. Each part may be approximately cylindrical in shape and made of different elastic materials. Even in man's own body, osseous tissue, in general, consists of two parts bonded end to end. One is cancellous (spongy) and the other is cortical bone, both may be approximately cylindrical in shape and are elastic in nature. Moreover, implantation of cylindrical bone with appropriate metal like Titanium results in a composite cylinder consisting of two cylinders of different materials bonded end to end. Excess stresses and pressure in the above elements result in vibrations. Plane strain vibrations of thick-walled hollow elastic cylinders were studied by Gazis (1957). Investigation of axisymmetric wave propagation in a two layered elastic

*For correspondence 
cylinder is made by Whitter \& Jones (1982). Kumar (1964) studied the propagation of axially symmetric waves in a finite elastic cylinder. Flexural vibrations of finite circular elastic cylinder were studied by Biswas et al (1976). Employing Biot's theory (Biot 1956), Tajuddin \& Sarma $(1978,1980)$ studied the torsional vibrations of finite hollow poroelastic cylinders. Torsional vibrations of finite composite poroelastic cylinders, either concentric or bonded end to end are investigated by Tajuddin (1982). Malla Reddy \& Tajuddin (2000) investigated plane-strain vibrations of thick-walled hollow poroelastic cylinders and discussed the extreme limit cases of plate and solid cylinder. Effect of boundaries on the dynamic interaction of a liquid-filled porous layer has been studied by Tajuddin \& Narayan Reddy (2005). Tajuddin \& Shah $(2006,2007)$ studied the circumferential waves and torsional vibrations of infinite hollow poroelastic cylinders. Axially symmetric vibrations of finite composite poroelastic cylinders that are bonded end to end are investigated by Shah \& Tajuddin (2009). The analysis of the flexural vibrations in cylindrical structures has wide applications in the field of acoustics structural design and Biomechanics, where the knowledge of natural mode of vibration is of paramount importance. There should be stress-free conditions in order to obtain natural modes theoretically. However, to the best of author's knowledge, flexural vibrations of finite composite poroelastic cylinder that consisting of two parts bonded end to end are not investigated. Therefore, in this paper, an attempt is made to investigate the same in the framework of Biot's theory. Frequency equations are obtained for both pervious surface and impervious surface. Phase velocities against two aspect ratios namely ratio of individual lengths of cylinders, and the ratio of the length of cylinder-I to radius are investigated.

This paper is organized as follows. In section 2, geometry of the problem is described. In section 3, basic governing equations, formulation, and solution of the problem are given. In

section 4, frequency equations are derived for both pervious and impervious surfaces. Some particular cases are derived in section 5, while numerical results are presented in section 6 . Finally, conclusions are given in section 7.

\section{Geometry of the problem}

Let $(r, \theta, z)$ be the cylindrical polar coordinates. Consider a composite isotropic poroelastic solid cylinder of length $L+c$ and radius $r_{1}$ whose axis is in the direction of $z$-axis. The cylinder is made of two cylinders of different poroelastic materials bonded at the plane ends. Let these be designated as cylinder-I and cylinder-II. Cylinder-I is of length $L$, while cylinder-II is of length $c$ and each of radius $r_{1}$. Let these cylinders are bonded at the plane ends at $z=0$ and other ends lie at $z=L$ and $z=-c$ as shown in figure 1 .

\section{Governing equations and solution of the problem}

The equations of motion of a homogeneous, isotropic poroelastic solid (Biot 1956) in the presence of dissipation $(b)$ are

$$
\begin{aligned}
N \nabla^{2} \vec{u}+(A+N) \nabla e+Q \nabla \varepsilon & =\frac{\partial^{2}}{\partial t^{2}}\left(\rho_{11} \vec{u}+\rho_{12} \vec{U}\right)+b \frac{\partial}{\partial t}(\vec{u}-\vec{U}), \\
Q \nabla e+R \nabla \varepsilon & =\frac{\partial^{2}}{\partial t^{2}}\left(\rho_{12} \vec{u}+\rho_{22} \vec{U}\right)-b \frac{\partial}{\partial t}(\vec{u}-\vec{U}),
\end{aligned}
$$




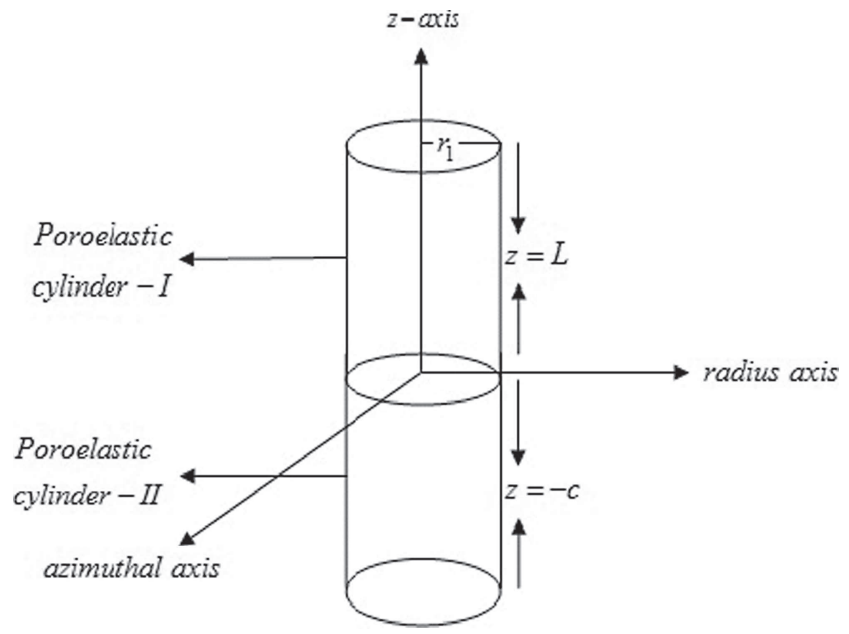

Figure 1. Finite composite poroelastic solid cylinder.

where $\nabla^{2}$ is Laplace operator, $\vec{u}(u, v, w)$ and $\vec{U}(U, V, W)$ are solid and fluid displacements, $e(=\nabla \cdot \vec{u})$ and $\varepsilon(=\nabla \cdot \vec{U})$ are the dilatations of solid and fluid, respectively; $A, N, Q, R$ are all poroelastic constants; $\rho_{i j}$ are mass coefficients. The relevant solid stresses $\sigma_{i j}$, fluid pressure $s$ and strain components are

$$
\begin{aligned}
\sigma_{i j} & =2 N e_{i j}+(A e+Q \varepsilon) \delta_{i j} \quad(i, j=1,2,3) \\
s & =Q e+R \varepsilon . \\
e_{r r} & =\frac{\partial u}{\partial r}, \quad e_{\theta \theta}=\frac{1}{r}\left(\frac{\partial v}{\partial \theta}+u\right), \quad e_{z z}=\frac{\partial w}{\partial z} \\
e_{r \theta} & =\frac{1}{2}\left(\frac{1}{r} \frac{\partial u}{\partial \theta}-\frac{v}{r}+\frac{\partial v}{\partial r}\right), \quad e_{r z}=\frac{1}{2}\left(\frac{\partial u}{\partial z}+\frac{\partial w}{\partial r}\right), \quad e_{\theta z}=\frac{1}{2}\left(\frac{\partial v}{\partial z}+\frac{1}{r} \frac{\partial w}{\partial \theta}\right) .
\end{aligned}
$$

In Eq. (2), $\delta_{i j}$ is the well-known Kronecker delta function. Poroelastic constants of cylinder-I and cylinder-II are denoted by $P, N, Q, R$ and $P^{*}, N^{*}, Q^{*}, R^{*}$, respectively. We introduce the displacement potentials $\phi$ 's and $\psi$ 's which are functions of $r, \theta, z$ and $t$ as follows:

$$
\begin{aligned}
\vec{u} & =\nabla \phi_{1}+\nabla \times \vec{\psi}_{1}, \\
\vec{U} & =\nabla \phi_{2}+\nabla \times \vec{\psi}_{2},
\end{aligned}
$$

where $\phi_{1}, \phi_{2}$ are scalars and $\vec{\psi}_{1}=\left(h_{r}, h_{\theta}, h_{z}\right), \quad \vec{\psi}_{2}=\left(H_{r}, H_{\theta}, H_{z}\right)$, which led to the following equations

$$
\begin{gathered}
u=\frac{\partial \phi_{1}}{\partial r}+\frac{1}{r} \frac{\partial h_{z}}{\partial \theta}-\frac{\partial h_{\theta}}{\partial z}, \quad v=\frac{1}{r} \frac{\partial \phi_{1}}{\partial \theta}+\frac{\partial h_{r}}{\partial z}-\frac{\partial h_{z}}{\partial r}, \quad w=\frac{\partial \phi_{1}}{\partial z}+\frac{\partial h_{\theta}}{\partial r}+\frac{h_{\theta}}{r}-\frac{1}{r} \frac{\partial h_{r}}{\partial \theta}, \\
U=\frac{\partial \phi_{2}}{\partial r}+\frac{1}{r} \frac{\partial H_{z}}{\partial \theta}-\frac{\partial H_{\theta}}{\partial z}, V=\frac{1}{r} \frac{\partial \phi_{2}}{\partial \theta}+\frac{\partial H_{r}}{\partial z}-\frac{\partial H_{z}}{\partial r}, W=\frac{\partial \phi_{2}}{\partial z}+\frac{\partial H_{\theta}}{\partial r}+\frac{H_{\theta}}{r}-\frac{1}{r} \frac{\partial H_{r}}{\partial \theta} .
\end{gathered}
$$


Let

$$
\begin{array}{rlrl}
\phi_{1}=f_{1}(r) \cos \theta e^{i(k z+\omega t)}, & \phi_{2}=f_{2}(r) \cos \theta e^{i(k z+\omega t)}, \\
h_{r}=g_{r}(r) \sin \theta e^{i(k z+\omega t)}, & & H_{r}=G_{r}(r) \sin \theta e^{i(k z+\omega t)}, \\
h_{\theta}=g_{\theta}(r) \cos \theta e^{i(k z+\omega t)}, & & H_{\theta}=G_{\theta}(r) \cos \theta e^{i(k z+\omega t)}, \\
h_{z}=g_{3}(r) \sin \theta e^{i(k z+\omega t)}, & & H_{z}=G_{3}(r) \sin \theta e^{i(k z+\omega t)},
\end{array}
$$

In Eq. (5), $k$ is a wave number, $\omega$ is the frequency, $i$ is the complex unity, and $t$ is time. Using Eq. (3), the equations of motion (Biot 1956) in terms of displacement potential functions are

$$
\begin{aligned}
P \nabla^{2} \phi_{1}+Q \nabla^{2} \phi_{2} & =\left(\rho_{11} \ddot{\phi}_{1}+\rho_{12} \ddot{\phi}_{2}\right)+b\left(\dot{\phi}_{1}-\dot{\phi}_{2}\right), \\
Q \nabla^{2} \phi_{1}+R \nabla^{2} \phi_{2} & =\left(\rho_{12} \ddot{\phi}_{1}+\rho_{22} \ddot{\phi}_{2}\right)-b\left(\dot{\phi}_{1}-\dot{\phi}_{2}\right), \\
N\left(\nabla^{2} h_{r}-\frac{h_{r}}{r^{2}}-\frac{2}{r^{2}} \frac{\partial h_{\theta}}{\partial \theta}\right) & =\left(\rho_{11} \ddot{h}_{r}+\rho_{12} \ddot{H}_{r}\right)+b\left(\dot{h}_{r}-\dot{H}_{r}\right), \\
N\left(\nabla^{2} h_{\theta}-\frac{h_{\theta}}{r^{2}}+\frac{2}{r^{2}} \frac{\partial h_{r}}{\partial \theta}\right) & =\left(\rho_{11} \ddot{h}_{\theta}+\rho_{12} \ddot{H}_{\theta}\right)+b\left(\dot{h}_{\theta}-\dot{H}_{\theta}\right), \\
N \nabla^{2} h_{z} & =\left(\rho_{11} \ddot{h}_{z}+\rho_{12} \ddot{H}_{z}\right)+b\left(\dot{h}_{z}-\dot{H}_{z}\right), \\
0 & =\left(\rho_{12} \ddot{h}_{r}+\rho_{22} \ddot{H}_{r}\right)-b\left(\dot{h}_{r}-\dot{H}_{r}\right), \\
0 & =\left(\rho_{12} \ddot{h}_{\theta}+\rho_{22} \ddot{H}_{\theta}\right)-b\left(\dot{h}_{\theta}-\dot{H}_{\theta}\right), \\
0 & =\left(\rho_{12} \ddot{h}_{z}+\rho_{22} \ddot{H}_{z}\right)-b\left(\dot{h}_{z}-\dot{H}_{z}\right),
\end{aligned}
$$

where dot over a quantity represents differentiation with respect to time $t$ and $P=A+2 N$. Eq. (6) with the help of Eq. (5) is reduced to

$$
\begin{aligned}
P \Delta f_{1}+Q \Delta f_{2} & =-\omega^{2}\left(K_{11} f_{1}+K_{12} f_{2}\right), \\
Q \Delta f_{1}+R \Delta f_{2} & =-\omega^{2}\left(K_{12} f_{1}+K_{22} f_{2}\right), \\
N\left(\Delta g_{r}-\frac{g_{r}}{r^{2}}+\frac{2 g_{\theta}}{r^{2}}\right) & =-\omega^{2}\left(K_{11} g_{r}+K_{12} G_{r}\right), \\
N\left(\Delta g_{\theta}-\frac{g_{\theta}}{r^{2}}+\frac{2 g_{r}}{r^{2}}\right) & =-\omega^{2}\left(K_{11} g_{\theta}+K_{12} G_{\theta}\right), \\
N\left(\Delta g_{3}\right) & =-\omega^{2}\left(K_{11} g_{3}+K_{12} G_{3}\right), \\
0 & =-\omega^{2}\left(K_{12} g_{r}+K_{22} G_{r}\right), \\
0 & =-\omega^{2}\left(K_{12} g_{\theta}+K_{22} G_{\theta}\right), \\
0 & =-\omega^{2}\left(K_{12} g_{3}+K_{22} G_{3}\right),
\end{aligned}
$$

where

$$
\Delta=\frac{d^{2}}{d r^{2}}+\frac{1}{r} \frac{d}{d r}-\frac{1}{r^{2}}-k^{2}, \quad K_{11}=\rho_{11}-\frac{i b}{\omega}, \quad K_{12}=\rho_{12}+\frac{i b}{\omega}, \quad K_{22}=\rho_{22}-\frac{i b}{\omega} .
$$

The general solutions of Eq. (7) can be obtained in terms of the Bessel function of first kind $J_{n}$. Equation (7) after a long calculation yields

$$
\begin{aligned}
f_{1} & =C_{1} J_{1}\left(\alpha_{1} r\right)+C_{2} J_{1}\left(\alpha_{2} r\right) \\
f_{2} & =-\left(C_{1} \delta_{1}^{2} J_{1}\left(\alpha_{1} r\right)+C_{2} \delta_{2}^{2} J_{1}\left(\alpha_{2} r\right)\right), \\
g_{3}(r) & =C_{3} J_{1}\left(\alpha_{3} r\right), \\
2 g_{1}(r) & =g_{r}-g_{\theta}=2 C_{4} J_{2}\left(\alpha_{3} r\right) \\
2 g_{2}(r) & =g_{r}+g_{\theta}=2 C_{5} J_{0}\left(\alpha_{3} r\right) .
\end{aligned}
$$


where

$$
\begin{gathered}
\alpha_{i}^{2}=\frac{\omega^{2}}{V_{i}^{2}}-k^{2}, \quad i=1,2,3 . \\
\delta_{i}^{2}=\frac{1}{R K_{11}-Q K_{22}}\left(\left(R K_{11}-Q K_{12}\right)-V_{i}^{-2}\left(P R-Q^{2}\right)\right), \quad i=1,2 .
\end{gathered}
$$

In Eq. (10), $V_{1}, V_{2}$ and $V_{3}$ are dilatational wave velocities of first and second kind, and shear wave velocity, respectively (Biot 1956). The gauge invariance property (Gazis 1959) is used to eliminate one integration constant from Eq. (9), accordingly, any one of the potential functions $g_{1}, g_{2}, g_{3}$ can be set equal to zero without loss of generality of the solution. Setting $g_{2}=0$, we can obtain

$$
g_{r}=-g_{\theta}=g_{1} \text {. }
$$

Substituting (5) in (4), the displacements of solid are

$$
\begin{aligned}
u & =\left(\frac{d f_{1}}{d r}+\frac{1}{r} g_{3}-i k g_{\theta}\right) \cos \theta e^{i(k z+\omega t)}, \quad v=\left(-\frac{1}{r} f_{1}+i k g_{r}-\frac{d g_{3}}{d r}\right) \sin \theta e^{i(k z+\omega t)}, \\
w & =\left(i k f_{1}+\frac{d g_{\theta}}{d r}+\frac{1}{r} g_{\theta}-\frac{1}{r} g_{r}\right) \cos \theta e^{i(k z+\omega t)} .
\end{aligned}
$$

Substituting Eq. (12) in Eq. (13), the displacement components become

$$
\begin{aligned}
u & =\left(\frac{d f_{1}}{d r}+\frac{1}{r} g_{3}+i k g_{1}\right) \cos \theta e^{i(k z+\omega t)}, \quad v=\left(-\frac{1}{r} f_{1}+i k g_{1}-\frac{d g_{3}}{d r}\right) \sin \theta e^{i(k z+\omega t)}, \\
w & =\left(i k f_{1}-\frac{d g_{1}}{d r}-\frac{2}{r} g_{1}\right) \cos \theta e^{i(k z+\omega t)} .
\end{aligned}
$$

For flexural vibrations, solid displacement components of cylinder-I can readily be evaluated from Eq. (14), which are given by

$$
\left[\begin{array}{c}
u \\
v \\
w
\end{array}\right]=\left[\begin{array}{llll}
A_{11}(r) \cos \theta & A_{12}(r) \cos \theta & A_{13}(r) \cos \theta & A_{14}(r) \cos \theta \\
A_{21}(r) \sin \theta & A_{22}(r) \sin \theta & A_{23}(r) \sin \theta & A_{24}(r) \sin \theta \\
A_{31}(r) \cos \theta & A_{32}(r) \cos \theta & A_{33}(r) \cos \theta & A_{34}(r) \cos \theta
\end{array}\right]\left[\begin{array}{l}
C_{1} \\
C_{2} \\
C_{3} \\
C_{4}
\end{array}\right] e^{i(k z+\omega t)}
$$

In Eq. (15), $C_{1}, C_{2}, C_{3}, C_{4}$ are all arbitrary constants and

$$
\begin{aligned}
& A_{11}(r)=\frac{1}{r} J_{1}\left(\alpha_{1} r\right)-\alpha_{1} J_{2}\left(\alpha_{1} r\right), \quad A_{12}(r)=\frac{1}{r} J_{1}\left(\alpha_{2} r\right)-\alpha_{2} J_{2}\left(\alpha_{2} r\right), \\
& A_{13}(r)=\frac{1}{r} J_{1}\left(\alpha_{3} r\right), \quad A_{14}(r)=i k J_{2}\left(\alpha_{3} r\right), \quad A_{21}(r)=\frac{-1}{r} J_{1}\left(\alpha_{1} r\right), \\
& A_{22}(r)=\frac{-1}{r} J_{1}\left(\alpha_{2} r\right), \quad A_{23}(r)=\frac{-1}{r} J_{1}\left(\alpha_{3} r\right)+\alpha_{3} J_{2}\left(\alpha_{3} r\right), \\
& A_{24}(r)=i k J_{2}\left(\alpha_{3} r\right), \quad A_{31}(r)=i k J_{1}\left(\alpha_{1} r\right), \quad A_{32}(r)=i k J_{1}\left(\alpha_{2} r\right), \\
& A_{33}(r)=0, \quad A_{34}(r)=-\alpha_{3} J_{1}\left(\alpha_{3} r\right),
\end{aligned}
$$

In the case of cylinder-II, the displacement components are

$$
\left[\begin{array}{c}
u^{*} \\
v^{*} \\
w^{*}
\end{array}\right]=\left[\begin{array}{llll}
A_{11}^{*}(r) \cos \theta & A_{12}^{*}(r) \cos \theta & A_{13}^{*}(r) \cos \theta & A_{14}^{*}(r) \cos \theta \\
A_{21}^{*}(r) \sin \theta & A_{22}^{*}(r) \sin \theta & A_{23}^{*}(r) \sin \theta & A_{24}^{*}(r) \sin \theta \\
A_{31}^{*}(r) \cos \theta & A_{32}^{*}(r) \cos \theta & A_{33}^{*}(r) \cos \theta & A_{34}^{*}(r) \cos \theta
\end{array}\right]\left[\begin{array}{c}
D_{1} \\
D_{2} \\
D_{3} \\
D_{4}
\end{array}\right] e^{i\left(k^{*} z+\omega t\right)} .
$$


In Eq. (17), $D_{1}, D_{2}, D_{3}, D_{4}$ are all arbitrary constants, $k^{*}$ is the wave number pertaining to cylinder-II, and $A_{i j}^{*}$ are the same as $A_{i j}$ with $\alpha_{i}^{2}$ are replaced by $\alpha_{i}^{* 2}$, where $\alpha_{i}^{* 2}=\frac{\omega^{2}}{V_{i}^{* 2}}-k^{* 2}$, $i=1,2,3$. The quantities $V_{1}^{*}, V_{2}^{*}$ and $V_{3}^{*}$ are dilatational wave velocities of first and second kind, and shear wave velocity in cylinder-II, respectively. By substituting the displacements in Eq. (2), the relevant stresses and fluid pressure pertaining to cylinder-I are

$$
\left[\begin{array}{c}
\sigma_{z z}+s \\
\sigma_{z \theta} \\
\sigma_{z r} \\
s
\end{array}\right]=\left[\begin{array}{llll}
M_{11}(r) \cos \theta & M_{12}(r) \cos \theta & M_{13}(r) \cos \theta & M_{14}(r) \cos \theta \\
M_{21}(r) \sin \theta & M_{22}(r) \sin \theta & M_{23}(r) \sin \theta & M_{24}(r) \sin \theta \\
M_{31}(r) \cos \theta & M_{32}(r) \cos \theta & M_{33}(r) \cos \theta & M_{34}(r) \cos \theta \\
M_{41}(r) \cos \theta & M_{42}(r) \cos \theta & M_{43}(r) \cos \theta & M_{44}(r) \cos \theta
\end{array}\right]\left[\begin{array}{c}
C_{1} \\
C_{2} \\
C_{3} \\
C_{4}
\end{array}\right] e^{i(k z+\omega t)}
$$

Fluid pressure gradient is

$$
\frac{\partial s}{\partial r}=\left(C_{1} N_{41}(r)+C_{2} N_{42}(r)\right) \cos \theta e^{i(k z+\omega t)} .
$$

In Eq. (18) and Eq. (19),

$$
\begin{aligned}
& M_{11}(r)=\left(\left((Q+R) \delta_{1}^{2}-(A+Q)\right) \alpha_{1}^{2}+\left((Q+R) \delta_{1}^{2}-(P+Q)\right) k^{2}\right) J_{1}\left(\alpha_{1} r\right), \\
& M_{12}(r)=\left(\left((Q+R) \delta_{2}^{2}-(A+Q)\right) \alpha_{2}^{2}+\left((Q+R) \delta_{2}^{2}-(P+Q)\right) k^{2}\right) J_{1}\left(\alpha_{2} r\right), \\
& M_{13}(r)=0, \quad M_{14}(r)=-2 N i k \alpha_{3} J_{1}\left(\alpha_{3} r\right), M_{21}(r)=-\frac{2 N i k}{r} J_{1}\left(\alpha_{1} r\right), \\
& M_{22}(r)=-\frac{2 N i k}{r} J_{1}\left(\alpha_{2} r\right), M_{23}(r)=-\left(\frac{N i k}{r} J_{1}\left(\alpha_{3} r\right)-i k N \alpha_{3} J_{2}\left(\alpha_{3} r\right)\right), \\
& M_{24}(r)=\frac{N \alpha_{3}}{r} J_{1}\left(\alpha_{3} r\right)-N k^{2} J_{2}\left(\alpha_{3} r\right), M_{31}(r)=\frac{2 N i k}{r} J_{1}\left(\alpha_{1} r\right)-2 N i k \alpha_{1} J_{2}\left(\alpha_{1} r\right), \\
& M_{32}(r)=\frac{2 N i k}{r} J_{1}\left(\alpha_{2} r\right)-2 N i k \alpha_{2} J_{2}\left(\alpha_{2} r\right), M_{33}(r)=\frac{N i k}{r} J_{1}\left(\alpha_{3} r\right), \\
& M_{34}(r)=-\left(\frac{N \alpha_{3}}{r} J_{1}\left(\alpha_{3} r\right)-N\left(\alpha_{3}^{2}-k^{2}\right) J_{2}\left(\alpha_{3} r\right)\right), \\
& M_{41}(r)=\left(R \delta_{1}^{2}-Q\right)\left(\alpha_{1}^{2}+k^{2}\right) J_{1}\left(\alpha_{1} r\right), \\
& M_{42}(r)=\left(R \delta_{2}^{2}-Q\right)\left(\alpha_{2}^{2}+k^{2}\right) J_{1}\left(\alpha_{2} r\right), M_{43}(r)=0, M_{44}(r)=0, \\
& N_{41}(r)=\left(R \delta_{1}^{2}-Q\right)\left(\alpha_{1}^{2}+k^{2}\right)\left(\frac{1}{r} J_{1}\left(\alpha_{1} r\right)-\alpha_{1} J_{2}\left(\alpha_{1} r\right)\right), \\
& N_{42}(r)=\left(R \delta_{2}^{2}-Q\right)\left(\alpha_{2}^{2}+k^{2}\right)\left(\frac{1}{r} J_{1}\left(\alpha_{2} r\right)-\alpha_{2} J_{2}\left(\alpha_{2} r\right)\right) .
\end{aligned}
$$

Similarly, the stresses and fluid pressure of cylinder-II are

$$
\begin{gathered}
{\left[\begin{array}{c}
\sigma_{z z}^{*}+s^{*} \\
\sigma_{z \theta}^{*} \\
\sigma_{z r}^{*} \\
s^{*}
\end{array}\right]=\left[\begin{array}{cccc}
M_{11}^{*}(r) \cos \theta & M_{12}^{*}(r) \cos \theta & M_{13}^{*}(r) \cos \theta & M_{14}^{*}(r) \cos \theta \\
M_{21}^{*}(r) \sin \theta & M_{22}^{*}(r) \sin \theta & M_{23}^{*}(r) \sin \theta & M_{24}^{*}(r) \sin \theta \\
M_{31}^{*}(r) \cos \theta & M_{32}^{*}(r) \cos \theta & M_{33}^{*}(r) \cos \theta & M_{34}^{*}(r) \cos \theta \\
M_{41}^{*}(r) \cos \theta & M_{42}^{*}(r) \cos \theta & M_{43}^{*}(r) \cos \theta & M_{44}^{*}(r) \cos \theta
\end{array}\right]\left[\begin{array}{c}
D_{1} \\
D_{2} \\
D_{3} \\
D_{4}
\end{array}\right] e^{i\left(k^{*} z+\omega t\right)}} \\
\frac{\partial s^{*}}{\partial r}=\left(D_{1} N_{41}^{*}(r)+D_{2} N_{42}^{*}(r)\right) \cos \theta e^{i\left(k^{*} z+\omega t\right)} .
\end{gathered}
$$


In Eqs. (21) and (22), $M_{i j}^{*}$ and $N_{i j}^{*}$ are the same as $M_{i j}$ and $N_{i j}$, respectively, but with the pertinent quantities and material parameters that are superscripted.

\section{Boundary conditions and frequency equations}

The extreme plane ends of the finite composite poroelastic cylinder are assumed to be free from the stresses and there is a perfect bonding at the interface. Thus the boundary conditions for finite composite poroelastic solid circular cylinder, in the case of pervious surface are

$$
\begin{gathered}
\left(\sigma_{z z}+s\right)-\left(\sigma_{z z}^{*}+s^{*}\right)=0, \quad \sigma_{z \theta}-\sigma_{z \theta}^{*}=0, \quad \sigma_{z r}-\sigma_{z r}^{*}=0 \\
s=0, \quad s^{*}=0 \\
u-u^{*}=0, \quad v-v^{*}=0, \quad w-w^{*}=0 \text { at } \quad z=0,0 \leq r \leq r_{1} \quad \forall r \\
\text { and } \sigma_{z z}+s=0 \text { at } z=L \text { and } \sigma_{z z}^{*}+s^{*}=0 \text { at } z=-c .
\end{gathered}
$$

In the case of impervious surface, the boundary conditions are the same as that of pervious surface except Eq. (24) on fluid pressure. Here we have

$$
\frac{\partial s}{\partial r}=0, \quad \frac{\partial s^{*}}{\partial r}=0 \text { at } z=0, \quad 0 \leq r \leq r_{1} \quad \forall r
$$

Equations (23)-(25) result in a system of eight equations in eight arbitrary constants $C_{1}, C_{2}, C_{3}, C_{4}, D_{1}, D_{2}, D_{3}$ and $D_{4}$. A nontrivial solution can be obtained if the determinant of the coefficients vanishes. Accordingly, we obtain the following frequency equation for a pervious surface:

$$
\left|p_{i j}\left(r_{1}\right)\right|=0, \quad(i, j=1,2,3 \ldots, 8)
$$

where

$$
p_{i j}\left(r_{1}\right)= \begin{cases}M_{i j}\left(r_{1}\right) & (i, j=1,2,3,4) \\ -M_{i(j-4)}^{*}\left(r_{1}\right) & (i=1,2,3, j=5,6,7,8) \\ 0 & (i=4, j=5,6,7,8) \\ 0 & (i=5, j=1,2,3,4) \\ M_{(i-1)(j-4)}^{*}\left(r_{1}\right) & (i=5, j=5,6,7,8) \\ A_{(i-5) j}\left(r_{1}\right) & (i=6,7,8, j=1,2,3,4) \\ -A_{(i-5)(j-4)}^{*}\left(r_{1}\right) & (i=6,7,8, j=5,6,7,8) .\end{cases}
$$

In the case of an impervious surface, the frequency equation is

$$
\left|q_{i j}\left(r_{1}\right)\right|=0, \quad(i, j=1,2,3 \ldots, 8),
$$

where

$$
q_{i j}\left(r_{1}\right)= \begin{cases}p_{i j}\left(r_{1}\right) & (i=1,2,3,6,7,8, j=1,2, \ldots .8) \\ N_{i j}\left(r_{1}\right) & (i=4, j=1,2) \\ 0 & (i=4, j=3,4,5,6,7,8) \\ 0 & (i=5, j=1,2,3,4,7,8) \\ N_{(i-1)(j-4)}^{*}\left(r_{1}\right) & (i=5, j=5,6) .\end{cases}
$$


Equation (26) of the boundary conditions gives

$$
k=\frac{(2 q+1) \pi}{L} \text { or } \frac{2 q \pi}{L}, q=0,1,2,3 \ldots \text { and } k^{*}=\frac{(2 q+1) \pi}{c} \text { or } \frac{2 q \pi}{c}, q=0,1,2,3 \ldots
$$

\section{Particular cases}

When the wavelength is infinite or the wave number is zero (that is, when $k=k^{*}=0$ ), the frequency equation in the case of pervious surface reduces to

$$
A_{1} A_{2}=0
$$

where $A_{1}$ is $6 \times 6$ determinant pertaining to extensional vibrations and is given by

$$
A_{1}=\left|\tilde{p}_{i j}\left(r_{1}\right)\right|, \quad(i=1,4,5,6,7,8 \text { and } j=1,2,3,5,6,7)
$$

$\tilde{p}_{i j}$ 's are same as $p_{i j}$ 's with $k=k^{*}=0$ and $A_{2}$ is $2 \times 2$ determinant pertaining to shear vibrations and is given by

$$
A_{2}=\left|\begin{array}{cc}
J_{1}\left(\alpha_{3} r_{1}\right) & J_{1}\left(\alpha_{3}^{*} r_{1}\right) \\
\alpha_{3} J_{2}\left(\alpha_{3} r_{1}\right) & \alpha_{3}^{*} J_{2}\left(\alpha_{3}^{*} r_{1}\right)
\end{array}\right| .
$$

In the case of an impervious surface, we can see that

$$
A_{3} A_{2}=0
$$

where $A_{3}$ is $6 \times 6$ determinant pertaining to extensional vibrations and is given by

$$
A_{3}=\left|\tilde{q}_{i j}\left(r_{1}\right)\right|, \quad(i=1,4,5,6,7,8 \text { and } j=1,2,3,5,6,7)
$$

In Eq. (37), $\tilde{q}_{i j}$ 's are the same as $q_{i j}$ 's with $k=k^{*}=0$.

From Eqs. (33) and (36), we can infer that shear vibrations are independent of nature of the surface when $k=k^{*}=0$. If the angular co-ordinate $\theta$ is made to be zero, the results of axially symmetric vibrations are obtained as a special case (Shah \& Tajuddin 2009).

\section{Numerical results}

Due to dissipative nature of the medium, waves are attenuated. Attenuation presents some difficulty in the definition of phase velocity; therefore, the case $b=0$ is considered for the numerical results. Even if we make $b$ zero, problem would be poroelastic in nature as the coefficients $A, N, Q, R$ would not vanish, only thing is mass coefficients $K_{i j}$ would be real. The following non-dimensional parameters are introduced to investigate the frequency equations:

$$
\begin{aligned}
a_{1} & =\frac{P}{H}, \quad a_{2}=\frac{Q}{H}, \quad a_{3}=\frac{R}{H}, \quad a_{4}=\frac{N}{H}, \\
d_{1} & =\frac{\rho_{11}}{\rho}, \quad d_{2}=\frac{\rho_{12}}{\rho}, \quad d_{3}=\frac{\rho_{22}}{\rho}, \\
\tilde{x} & =\left(\frac{V_{0}}{V_{1}}\right)^{2}, \quad \tilde{y}=\left(\frac{V_{0}}{V_{2}}\right)^{2}, \quad \tilde{z}=\left(\frac{V_{0}}{V_{3}}\right)^{2},
\end{aligned}
$$




$$
\begin{aligned}
b_{1} & =\frac{P^{*}}{H}, \quad b_{2}=\frac{Q^{*}}{H}, \quad b_{3}=\frac{R^{*}}{H}, \quad b_{4}=\frac{N^{*}}{H}, \\
g_{1} & =\frac{\rho_{11}^{*}}{\rho}, \quad g_{2}=\frac{\rho_{12}^{*}}{\rho}, \quad g_{3}=\frac{\rho_{22}^{*}}{\rho}, \\
\tilde{x}^{*} & =\left(\frac{V_{0}}{V_{1}^{*}}\right)^{2}, \quad \tilde{y}^{*}=\left(\frac{V_{0}}{V_{2}^{*}}\right)^{2}, \quad \tilde{z}^{*}=\left(\frac{V_{0}}{V_{3}^{*}}\right)^{2}, \\
m & =\frac{d}{c_{0}}, \quad d=\frac{\omega}{k}, \quad R_{0}=\frac{L}{r_{1}}, \quad R_{1}=\frac{L}{c} .
\end{aligned}
$$

In Eq. (38), $d$ is the phase velocity, $m$ is the non-dimensional phase velocity, $R_{0}$ is ratio of the length of cylinder-I to radius, and $R_{1}$ is the ratio of lengths of the cylinders, $H=P+2 Q+R$ and $\rho=\rho_{11}+2 \rho_{12}+\rho_{22}$. Also, $c_{0}$ and $V_{0}$ are reference velocities and are given by $c_{0}^{2}=\frac{N}{\rho}$,

$V_{0}^{2}=\frac{H}{\rho}$. Employing these non-dimensional quantities in the frequency equations, we obtain implicit relations between non-dimensional phase velocity $(m), R_{0}$ and $R_{1}$. For illustration purpose, two cases are considered, one is sandstone composite cylinder, and the other is cylindrical bone implanted with Titanium.

\subsection{Sandstone composite cylinders}

Non-dimensional phase velocity $(m)$ is computed for both composite poroelastic cylinders, composite cylinder-I and composite cylinder-II for $q=1$. Numerical process is performed on MATLAB. The composite cylinder-I consists of the cylinder of the length $L$ made up of sandstone saturated with water (Fatt 1957), and the cylinder of length $c$ made up of sandstone saturated with kerosene (Yew \& Jogi 1976), while in composite cylinder-II, the materials are reversed. The physical parameters of these composite cylinders following Eq. (38) are given in table 1 . The numerical values are depicted in figures $2-5$. Figures $2-4$ depict variation of phase velocity in composite cylinder-I and composite cylinder-II against the ratio of lengths of cylinders $\left(R_{1}\right)$ when $R_{0}=0.5,1$, and 2 respectively, for the cases of pervious and impervious surfaces. In all the cases, curves are periodic in nature. From figures $2-4$, it is clear that the values of cylinder-I are, in general, greater than that of cylinder-II in the case of both pervious and impervious surfaces. Also, the values of pervious surface are, in general, greater than that of impervious surface for both cylinders. The nature of surface is causing this discrepancy. In the case of axially symmetric problem with the same geometry (Shah \& Tajuddin 2009), the trend is reversed, that is, the values of impervious surface are greater than that of pervious surface. Flexural nature might be responsible for this discrepancy. From figure 4, it is found that the values of pervious surface and impervious surface are close to each other for the cylinder-II beyond the point $R_{1}=1.2$. That is, in the case of cylinder-II, nature of the surface does not influence the values beyond the point $R_{1}=1.2$. But, in the same region, the nature of surface matters in the case of cylinder-I. In all the cases, the values are higher when the ratio of lengths of cylinder $\left(R_{1}\right)$ is lowest.

\subsection{Cylindrical bone implanted with Titanium}

If a cylindrical bone is implanted with Titanium at the plane end of the bone, then we have a composite cylinder consisting of two different materials one is bone and the other is Titanium. The natural selection of Titanium is obvious for its favourable characteristics including immunity to corrosion, bio-compatibility, and the capacity for joining with bone, which is 
Table 1. Material parameter values.

\begin{tabular}{lcc}
\hline Material parameter & Composite cylinder-I & Composite cylinder-II \\
\hline$a_{1}$ & 0.445 & 1.819 \\
$a_{2}$ & 0.034 & 0.011 \\
$a_{3}$ & 0.015 & 0.054 \\
$a_{4}$ & 0.123 & 0.780 \\
$d_{1}$ & 0.887 & 0.891 \\
$d_{2}$ & -0.001 & 0 \\
$d_{3}$ & 0.099 & 0.125 \\
$\tilde{x}$ & 1.863 & 0.489 \\
$\tilde{y}$ & 8.884 & 2.330 \\
$\tilde{z}$ & 7.183 & 1.142 \\
& & \\
$b_{1}$ & 0.96 & 0.843 \\
$b_{2}$ & 0.006 & 0.065 \\
$b_{3}$ & 0.028 & 0.028 \\
$b_{4}$ & 0.412 & 0.234 \\
$g_{1}$ & 0.887 & 0.901 \\
$g_{2}$ & 0 & -0.001 \\
$g_{3}$ & 0.123 & 0.101 \\
$\tilde{x}^{*}$ & 0.913 & 0.999 \\
$\tilde{y}^{*}$ & 4.347 & 4.763 \\
$\tilde{z}^{*}$ & 2.129 & 3.851 \\
\hline
\end{tabular}

osseointegration. Despite the said nature, there have been several reports of discoloration due to metal debris in the peri-implant tissue (Hamid Reza Seyyed Hosseinzadeh et al 2010). Titanium implants remain virtually unchanged in appearance, which offers high stiffness and strength, good ductility, and is usually well tolerated biologically. Its density is $4.5 \times 10^{3} \mathrm{~kg} / \mathrm{m}^{3}$. Its Young's modulus and Poisson ratio are $105 \mathrm{GPa}$ and 0.32 , respectively. Lame's constants, and thereby dilatational wave velocity and shear wave velocity are computed. Bone poroelastic parameter values $A, N, Q, R$ and mass coefficients $\rho_{i j}$

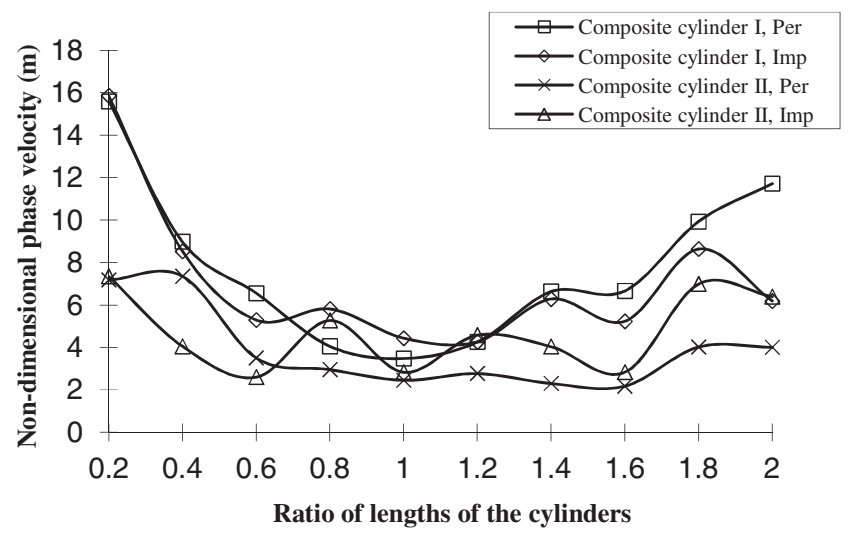

Figure 2. Variation of non-dimensional phase velocity with the ratio of lengths of cylinders when $R_{0}=0.5$. 


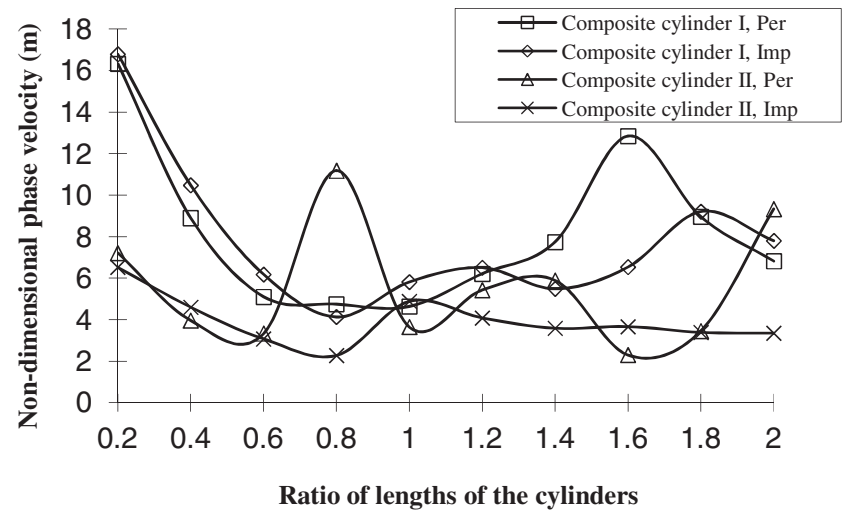

Figure 3. Variation of non-dimensional phase velocity with the ratio of lengths of cylinders when $R_{0}=1$.

are computed as in the paper (Nowinski \& Davis 1971). The values of Young's modulus and Poisson ratio are taken to be $20.684 \mathrm{GPa}$ and 0.28 , respectively as suggested in the paper (Nowinski \& Davis 1971). Mass coefficients of solid part and liquid part are taken to be $1.7634 \times 10^{3} \mathrm{~kg} / \mathrm{m}^{3}$ and $1.4961 \times 10^{2} \mathrm{~kg} / \mathrm{m}^{3}$, respectively, and mass coupling parameter is taken to be zero as in the paper (Nowinski \& Davis 1971). Velocities of dilatational waves and shear wave are computed. Non-dimensional phase velocity $(m)$ is computed against $R_{1}$, for fixed $R_{0}=0.5,1,2$ and the results are presented graphically in figure 5. From the figure, it is observed that the ratio of lengths of cylinder-I to radius $\left(R_{0}=\frac{L}{r_{1}}\right)$ does not have influence over phase velocity as $\left(R_{1}=\frac{L}{c}\right)$ increases, the said ratio has influence over phase velocity. That is, when the length of implanted titanium is relatively high then phase velocity is high consequently possibility of healing is low. In the neighborhood of 0.4 of $R_{1}$ the trend is stationary in all the three cases. When $R_{0}=1$, the values are nearly constant over the interval $(1.2,1.6)$ of $R_{1}$.

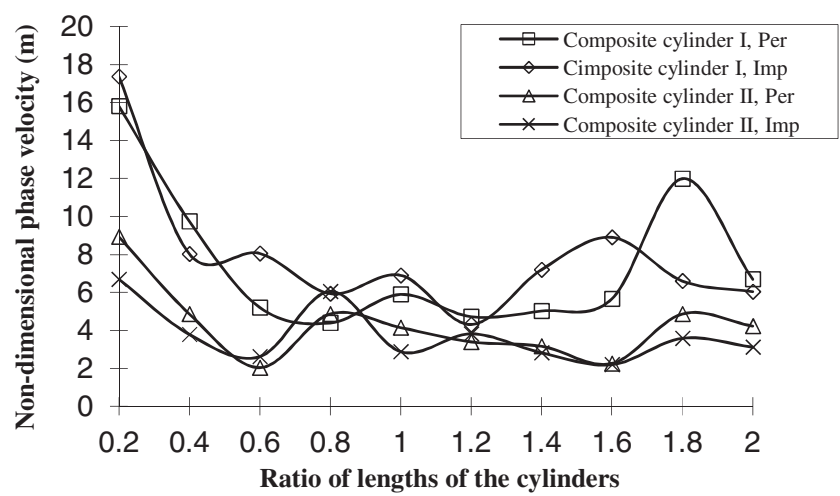

Figure 4. Variation of non-dimensional phase velocity with the ratio of lengths of cylinders when $R_{0}=2$. 


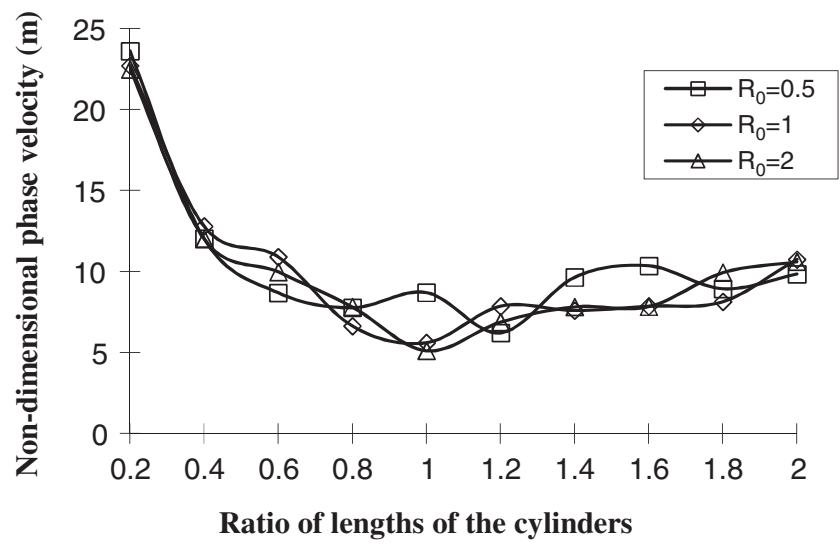

Figure 5. Variation of non-dimensional phase velocity with the ratio of lengths of cylinders in the case of bone implanted with Titanium.

\section{Conclusions}

Flexural vibrations in isotropic poroelastic composite cylinder consisting of two cylinders of different lengths that are bonded end to end are investigated in the framework of Biot's theory. It is clear from the analysis that nature of the surface does not have any influence over shear vibrations when wavelength is infinitely large. Phase velocity against the ratio of individual lengths of both the cylinders, and the ratio of the length of the cylinder-I to radius are investigated for two different composite cylinders and illustrated the conditions under which phase velocity values are greater. Similar analysis is made for a cylindrical bone when it is implanted with Titanium. The trend between implanted Titanium bone and sandstone cylinders is quite different. In the case of bone implanted Titanium, the phase velocity values are not so dispersed as that of composite sandstone cylinders as ratio of lengths of the cylinder-I to radius varies. From this analysis, we can assess the phase velocity values at given ratio of lengths of the cylinders and the ratio of lengths of cylinder-I to radius. This way we can assess the degree of degeneration. This kind of analysis can be made for any composite cylinder made of two different poroelastic solids if the values of all constants of pertinent solids are available.

\section{Acknowledgements}

One of the authors (Srisailam Aleti) would like to thank the University Grants Commission (UGC), Government of India for the funding through D.S. Kothari Postdoctoral Fellowship.

\section{List of symbols:}

$(r, \theta, z)$
$A, N, Q, R, A^{*}, N^{*}, Q^{*}, R^{*}$
$b$
$d$
$e$
$k, k^{*}$

cylindrical coordinate system

poroelastic constants

dissipation

phase velocity

solid dilatation

wave numbers 


$L+c$
$R_{0}$
$R_{1}$
$r_{1}$
$s, s^{*}$
$\vec{u}$
$\vec{U}$
$V_{1}, V_{2}, V_{3}, V_{1}^{*}, V_{2}^{*}, V_{3}^{*}$
$\varepsilon$
$m$
$\rho_{11}, \rho_{12}, \rho_{22}, \rho_{11}^{*}, \rho_{12}^{*}, \rho_{22}^{*}$
$\sigma_{i j}, \sigma_{i j}^{*}$
$\phi_{1}, \phi_{2}, \vec{\psi}_{1},, \vec{\psi}_{2}$
$\omega$
$\nabla^{2}$

length of the composite cylinder

ratio of length of cylinder-I to radius

ratio of lengths of cylinders

radius of the composite cylinder

fluid pressure

solid displacement

fluid displacement

dilatational wave velocities of first and second type,

shear wave velocity

fluid dilatation

non-dimensional phase velocity

mass coefficients

stresses

potential functions

frequency

Laplacian operator

\section{References}

Biot M A 1956 The theory of propagation of elastic waves in fluid-saturated porous solid. J. Acous. Soc. Am. 28: 168-178

Biswas R N, Nangia A K and Ram Kumar 1976 Flexural vibrations of a finite circular cylinder. Acoustic 35: $26-31$

Fatt I 1957 The Biot-Willis elastic coefficients for a sandstone. J. Appl. Mech. 26: 296-297

Gazis D C 1957 Exact analysis of plane-strain vibrations of thick-walled hollow cylinders. J. Acoust. Soc. Am. 30: 786-794

Gazis D C 1959 Three dimensional investigation of waves in hollow circular cylinders, I., Analytical Foundation. J. Acoust. Soc. Am. 31: 568-573

Hamid Reza Seyyed Hosseinzadeh M D, Mehrnoush Hassas Yeganeh M D and Hamed Reza Seyyed Hosseinzadeh 2010 Plate fixation in orthopaedics: Basic concepts and clinical applications

Malla Reddy P and Tajuddin M 2000 Exact analysis of the plane strain vibrations of thick walled hollow poroelastic cylinders. Int. J. Solids Struct. 37: 3439-3456

Nowinski J L and Davis C F 1971 Propagation of longitudinal waves in circularly cylindrical bone elements. Trans. ASME, J. Appl. Mech. 578-584

Ram Kumar 1964 Axially symmetric vibrations of a finite isotropic cylinder. J. Acoust. Soc. Am. 38: 851854

Shah S A and Tajuddin M 2009 Axially symmetric vibrations of finite composite poroelastic cylinder. Int. J. Appl. Mech. Eng. 14(3): 865-877

Tajuddin M 1982 Torsional vibrations of finite composite poroelastic cylinders. Indian J. Pure Appl. Math. 13: $375-381$

Tajuddin M and Ahmed Shah S 2006 Circumferential waves of infinite hollow poroelastic cylinders. Trans. ASME, J. Appl. Mech. 73: 705-708

Tajuddin M and Narayan Reddy G 2005 Effect of boundaries on the dynamic interaction of a liquid-filled porous layer and a supporting continuum. Sadhana 30(4): 527-535

Tajuddin M and Sarma K S 1978 Torsional vibrations of finite hollow poroelastic circular cylinders. Def. Sci. J. 28: 97-102

Tajuddin M and Sarma K S 1980 Torsional vibrations of poroelastic circular cylinders. Trans. ASME, J. Appl. Mech. 47: 214-216 
Tajuddin M and Shah S A 2007 On torsional vibrations of infinite hollow poroelastic cylinders. $J$. Mech. Mater Struct. 2(1): 189-200

Whitter J S and Jones J P 1982 Axially symmetric wave propagation in two layered cylinder. Int. J. Solids Struct. 3: 657-675

Yew C H and Jogi P N 1976 Study of wave motions in fluid-saturated porous rocks. J. Acoust. Soc. Am. 60: $2-8$ 